\title{
Two new Mallinella species from southern China (Araneae, Zodariidae)
}

\author{
Chi Jin ${ }^{1, \dagger}$, Feng Zhang ${ }^{1, \dagger}$ \\ I College of Life Sciences, Hebei University, Baoding, Hebei 071002, P. R. China \\ † urn:lsid:zoobank.org:author:5B2341AF-89E3-4B5E-A6CF-6FE323097182 \\ † urn:lsid:zoobank.org:author:904C4445-AAEA-40BC-B418-502053EF39B8 \\ Corresponding author: Feng Zhang (dudu06042001@163.com)
}

Academic editor: C. Rollard | Received 3 January 2013 | Accepted 19 April 2013 | Published 30 April 2013

urn:lsid:zoobank.org:pub:5B64F541-FFEB-4D87-9C2D-4746055B4854

Citation: Jin C, Zhang F (2013) Two new Mallinella species from southern China (Araneae, Zodariidae). ZooKeys 296: 79-88. doi: 10.3897/zookeys.296.4622

\begin{abstract}
Two new species of the spider genus Mallinella Strand, 1906 are reported from China: Mallinella sphaerica sp. n. (male, female) from Tianmu Mountain, Zhejiang Province and M. pluma sp. n. (male) from Daming Mountain, Guangxi Zhuang Autonomous Region.
\end{abstract}

\section{Keywords}

Spider, Mallinella, taxonomy, new species, China

\section{Introduction}

The spider family Zodariidae is represented by 78 genera and 1068 known species mainly from Africa, Australia and Asia (Platnick 2013), but only 36 species in 7 genera (Asceua Thorell, Cydrela Thorell, Mallinella Strand, Storenomorpha Simon, Heradion Dankittipakul \& Jocqué, Zodariellum Andreeva \& Tyschchenko and Heliconilla Pakawin et al.) have been reported from China (Platnick 2013; Zhang et al. 2012a, b; Dankittipakul et al. 2012).

The genus Mallinella was established by Strand in 1906, was redefined by Jocqué (1991) and differs from the other zodariids by the following characters: the sternum with

Copyright Chi Jin, Feng Zhang. This is an open access article distributed under the terms of the Creative Commons Attribution License 3.0 (CC-BY), which permits unrestricted use, distribution, and reproduction in any medium, provided the original author and source are credited. 
triangular extensions fitting in coxal concavities, the presence of a row of short spines in front of the tracheal spiracle, and a well-developed conductor which is a terminal excrescence of the tegulum (Jocqué, 1991). Until now, 202 Mallinella species have been reported worldwide (Platnick 2013), including 17 Mallinella species from southern China (Song and Kim 1997; Song et al. 1999; Wang et al. 1999; Yin and Yan 2001; Bao and Yin 2002; Zhang and Zhu 2009; Wang et al. 2009; Wang et al. 2009; Zhang et al. 2011, Zhang et al. 2012a; Dankittipakul et al. 2012).

While examining spiders collected from Tianmu Mountain, Zhejiang Province, and Daming Mountain, Guangxi Zhuang Autonomous Region of southern China, we found two species new to science - M. sphaerica sp. n. and M. pluma sp. n. that are described herein.

\section{Material and methods}

All specimens were kept in 75\% ethanol, examined and measured under a Tech XTL-II stereomicroscope. Drawings of M. sphaerica sp. n. were prepared under a Nikon SMZ 1500 stereomicroscope equipped with a drawing tube, and $M$. pluma sp. n. under a Leica M165C stereomicroscope equipped with a drawing tube. The photos were taken with a Leica M205A stereomicroscope equipped with a DFC450 CCD. Carapace length was measured from the anterior margin to the centre of the posterior margin. Eye size was measured as the maximum diameter of the lens in dorsal or frontal view. The leg measurements are presented as total length (femur, patella, tibia, metatarsus, tarsus). The epigyne was cleaned in a warm solution of potassium hydroxide $(\mathrm{KOH})$ and transferred to $75 \%$ ethanol for drawing. All measurements are in millimeter. All specimens studied are deposited in the Museum of Hebei University (MHBU), Baoding, China.

\section{Abbreviations}

$\begin{array}{ll}\text { ALE } & \text { anterior lateral eyes } \\ \text { AME } & \text { anterior median eyes } \\ \text { C } & \text { conductor } \\ \text { CD } & \text { copulatory ducts } \\ \text { E } & \text { embolus } \\ \text { EP } & \text { epigynal plate } \\ \text { MA } & \text { median apophysis } \\ \text { MOA } & \text { median ocular area } \\ \text { PLE } & \text { posterior lateral eyes } \\ \text { PME } & \text { posterior median eyes } \\ \text { RTA } & \text { retrolateral tibial apophysis } \\ \text { S } & \text { spermathecae } \\ \text { T } & \text { tegulum. }\end{array}$




\section{Taxonomy}

\section{Zodariidae Thorell, 1881}

\section{Mallinella sphaerica sp. $\mathbf{n}$.}

urn:lsid:zoobank.org:act:6591D5D0-F5D3-4498-8C74-3900F1A8270C

http://species-id.net/wiki/Mallinella_sphaerica

Figs 1-15

Type material. Holotype ${ }^{\lambda}$, CHINA, Zhejiang Province: Tianmu Mountain $\left(30^{\circ} 18^{\prime} \mathrm{N}\right.$, $119^{\circ} 27^{\prime}$ E), alt. 262m, Lin'an City, 25 July 2011, C. Jin leg. Paratype: 19 , same data as holotype.

Diagnosis. According to Dankittipakul et al. 2012, this species should belong to sub-group 3 of the fronto species-group. Males can be distinguished from these in other Mallinella species by the spherical median part of the median apophysis and the irregular rolled apex of the conductor in ventral view (Figs 10, 14). The female is extremely similar to that of $M$. cymbiforma Wang, Yin \& Peng, 2009, but differs from the latter by the thinner copulatory ducts (Figs 8, 13), the wider posterior margin of the epigynal plate $($ Figs 7,12 ), and MOA wider at the back (MOA wider at the front in M. cymbiforma).

Etymology. The specific name is a Latin adjective and refers to the spherical median part of the median apophysis.

Description. Male (holotype). Total length 6.27; prosoma 3.11 long, 2.14 wide; opisthosoma 2.96 long, 1.99 wide. Diameters of eyes: AME 0.20, ALE 0.15, PME 0.15, PLE 0.15. Distances between eyes: AME-AME 0.08, AME-ALE 0.10, ALEALE 0.55, PME-PME 0.13, PME-PLE 0.25, PLE-PLE 0.78, ALE-PLE 0.05. MOA 0.48 long, anterior 0.50 , posterior 0.43 . Clypeal height 0.75 . Labium 0.58 long, 0.50 wide. Sternum 1.35 long, 1.28 wide. Leg measurements: leg I 10.36 (2.55, 0.77, 2.35, 2.65, 2.04), II 9.90 (2.50, 0.92, 2.04, 2.60, 1.84), III 9.29 (2.35, 0.82, 1.94, 2.65, 1.53), IV 12.09 (2.70, 0.92, 2.60, 3.88, 1.99). Leg formula: 4123.

Carapace (Fig. 1) blackish-brown; fovea black, slightly swollen. Both eye rows (Fig. 3) procurved in dorsal view. Clypeus dark brown. Chelicerae dark brown, distally yellowish-brown. Endites yellowish-brown. Labium triangular, dark brown, distally yellowish-brown. Sternum brown and furnished with sparse black setae, lateral margin with small, pointed extensions fitting in coxal concavities. Legs brown, femora dark brown, coxae yellowish-brown; metatarsi II-IV distally with ventral hair tufts. Opisthosoma (Fig. 1) oval, longer than wide, dorsum with three pairs of white lateral patches (the third connected), followed by one transverse white patch centrally. Dorsal scutum reddish-brown, about half as long as opisthosoma. Venter grey-black, covered with three irregular longitudinal white stripes; posterior ventral spines thin (Fig. 5), arranged in a single row. Spinnerets pale yellow.

Palpal organ (Figs 9-11, 14, 15). RTA digitiform, slightly wider at base, gradually tapering towards pointed apex. Cymbial fold broad, approximately half as long as 


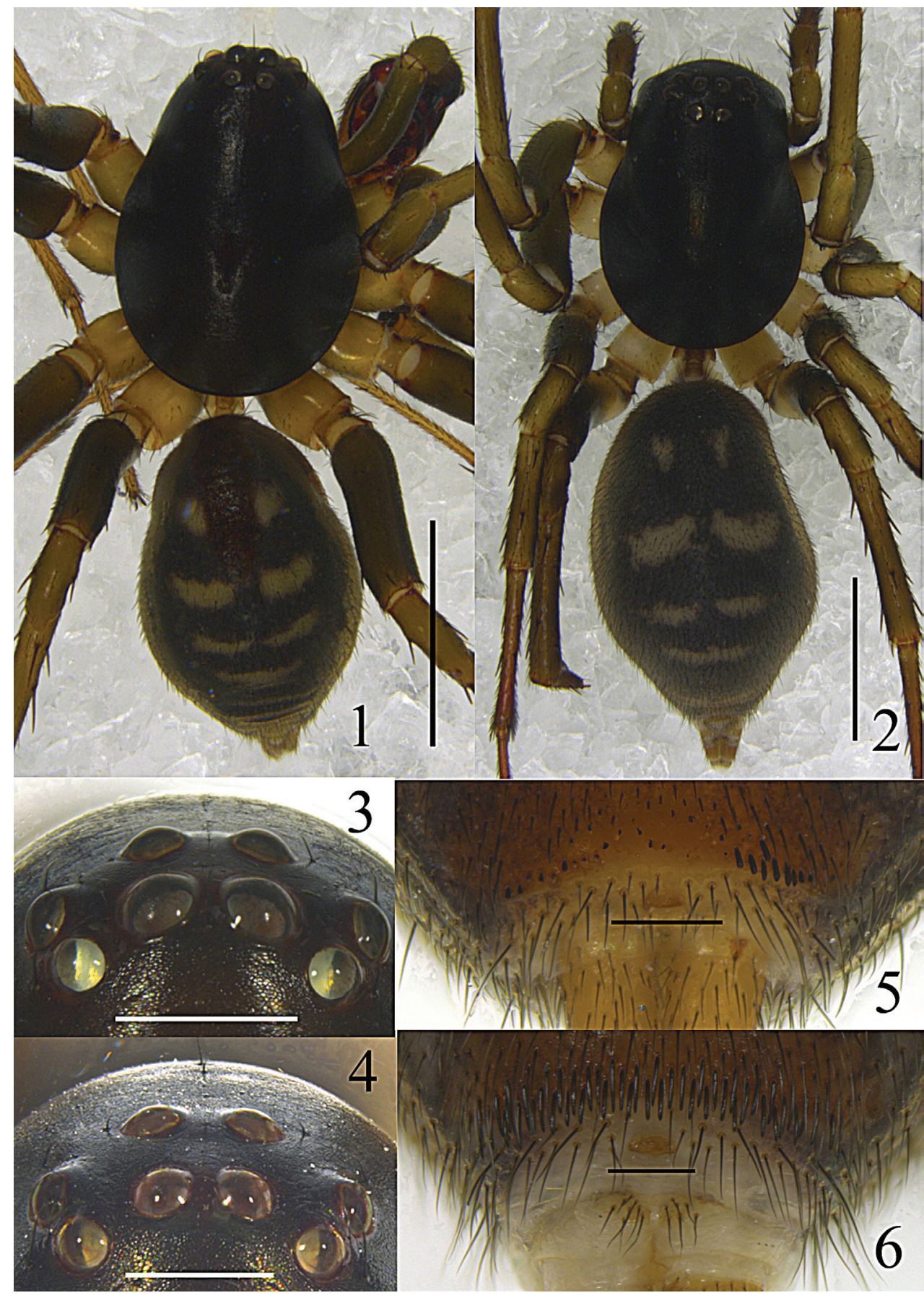

Figures I-6. Mallinella sphaerica sp. n., I male habitus, dorsal view $\mathbf{2}$ female habitus, dorsal view $\mathbf{3}$ male ocular area, frontal view $\mathbf{4}$ female, ocular area, frontal view $\mathbf{5}$ male, posterior ventral spines, ventral view 6 female, posterior ventral spines, ventral view. Scale bars: $2 \mathrm{~mm}(\mathbf{1}-\mathbf{2}) ; 0.5 \mathrm{~mm}(\mathbf{3}-\mathbf{4}) ; 0.2 \mathrm{~mm}$ (5-6). 


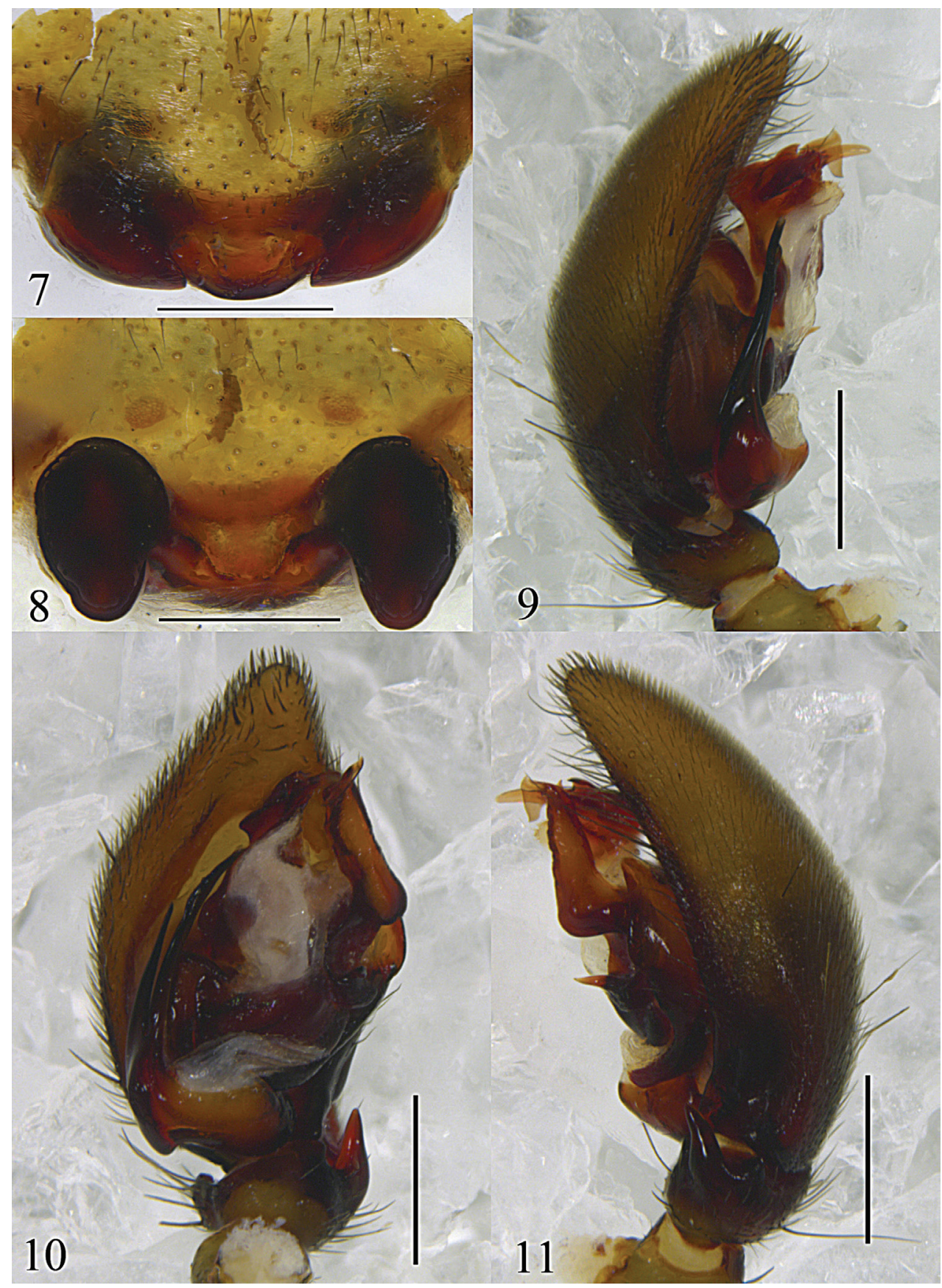

Figures 7-I I. Mallinella sphaerica sp. n., 7 epigyne, ventral view 8 vulva 9 left male palp, prolateral view I 0 same, ventral view II same, retrolateral view. Scale bars: $0.5 \mathrm{~mm}(7-\mathbf{1 1})$. 

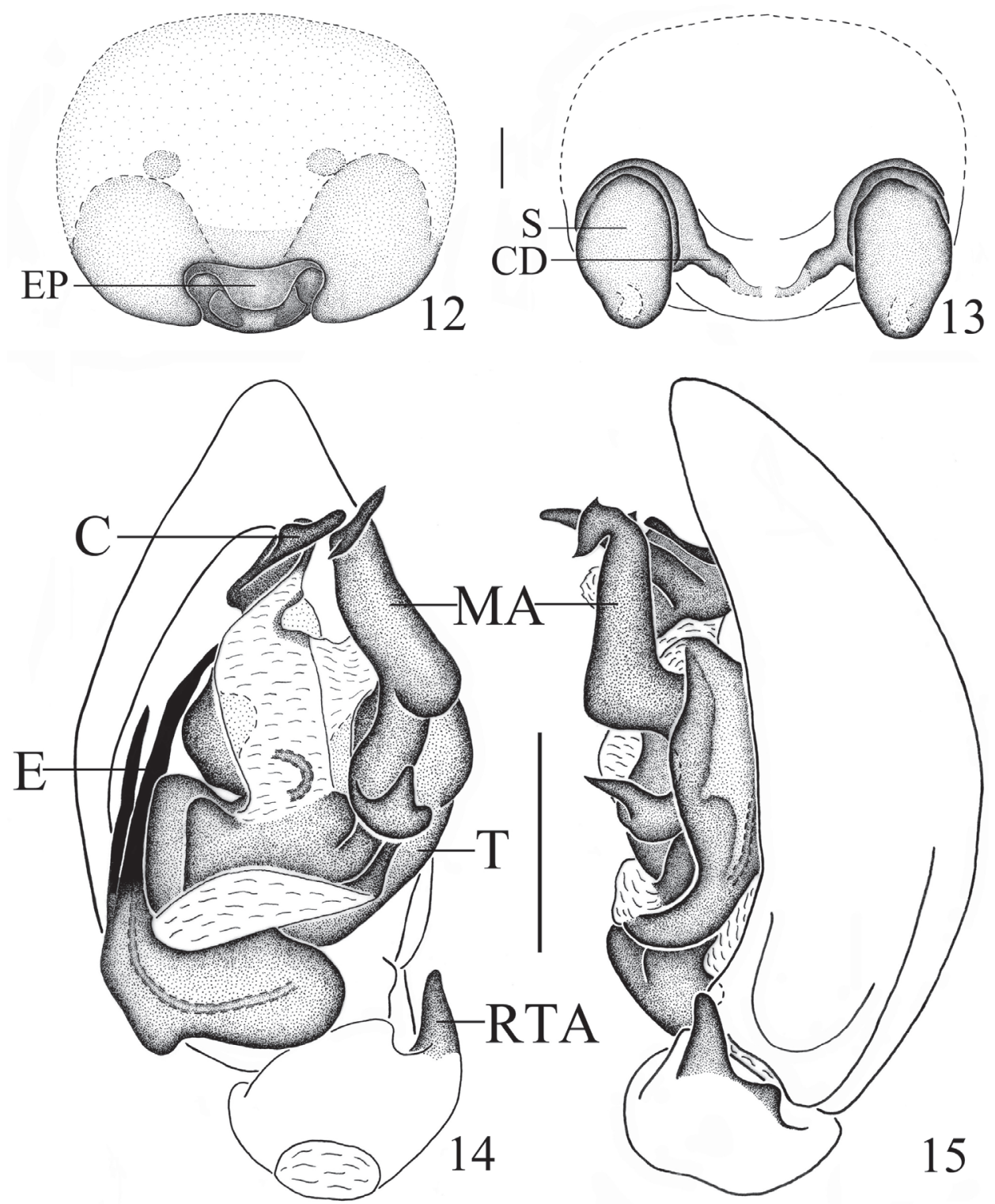

Figures I 2-15. Mallinella sphaerica sp. n., I 2 epigyne, ventral view I 3 vulva I 4 left male palp, ventral view 15 same, retrolateral view. Scale bars: $0.2 \mathrm{~mm}$ (12-13); $0.5 \mathrm{~mm}$ (14-15).

cymbium. Apex of median apophysis bifid, with sharply pointed tips on both ends; the median part of median apophysis spherical; baso-retrolateral fold narrow, triangular, apex pointed. Apex of conductor irregularly rolled. Embolus bifurcate, lateral ramus shorter than mesal ramus, with pointed apices.

Female (paratype). Total length 8.26; prosoma 3.57 long, 2.55 wide; opisthosoma 4.28 long, 2.75 wide. Diameters of eyes: AME 0.20, ALE 0.18, PME 0.18, PLE 0.18. 
Distances between eyes: AME-AME 0.08, AME-ALE 0.10, ALE-ALE 0.60, PMEPME 0.15, PME-PLE 0.28, PLE-PLE 0.88, ALE-PLE 0.05. MOA 0.53 long, anterior width 0.45 , posterior width 0.50 . Clypeal height 1.03 , Labium 0.63 long, 0.63 wide. Sternum 1.53 long, 1.53 wide. Leg measurements: leg I 8.68 (2.45, 0.97, 1.94, $1.79,1.53)$, II $8.47(2.19,0.97,1.79,1.99,1.53)$, III 8.00 (2.19, 0.92, 1.68, 2.14, 1.07), IV 10.34 (2.14, 1.02, 2.24, 3.06, 1.68). Leg formula: 4123.

Coloration and pattern (Fig. 2) as in male, but the color of coxae slightly paler than in male. Median furrow and scutum indistinct.

Epigyne-vulva (Figs 7, 8, 12, 13). Epigynal plate small, nearly reniform, with swollen posterior margin; copulatory openings hidden in a groove. Spermathecae large and oval; copulatory ducts thin; slender fertilization ducts invisible in dorsal view.

Distribution. China (Zhejiang).

\section{Mallinella pluma sp. n.}

urn:lsid:zoobank.org:act:0E7F143C-78AA-4CF0-A24D-9F1926101EB8

http://species-id.net/wiki/Mallinella_pluma

Figs 16-24

Type material. Holotype $\overbrace{}^{\lambda}$, CHINA, Guangxi, Zhuang Autonomous Region: Daming Mountain $\left(23^{\circ} 31^{\prime} \mathrm{N}, 108^{\circ} 21^{\prime} \mathrm{E}\right.$; 398m), Wuming County, Nanning City, 20 May 2011, Y. N. Wang leg.

Diagnosis. Male can be easily distinguished from other Mallinella males by the extremely narrow and long median apophysis (almost 2/3 cymbium length in ventral view), by the ventral row of plumose hairs near the tip of the cymbium (Figs 20, 22, 24), and also by the dense dorsal hair cover of the opisthosoma (Fig. 16).

Etymology. The specific name is a Latin noun and refers to the plumose hairs near the tip of cymbium.

Description. Male (holotype). Total length 5.30; prosoma 2.86 long, 2.04 wide; opisthosoma 2.24 long, 1.84 wide. Diameters of eyes: AME 0.18, ALE 0.15, PME 0.13, PLE 0.15. Distances between eyes: AME-AME 0.08, AME-ALE 0.10, ALEALE 0.50, PME-PME 0.13, PME-PLE 0.23, PLE-PLE 0.73, ALE-PLE 0.05. MOA 0.40 long, front width 0.40 , back width 0.38 . Clypeal height 0.80 . Labium 0.53 long, 0.48 wide. Sternum 1.28 long, 1.22 wide. Measurements of legs: leg I 9.51 (2.30, $0.77,2.24,2.35,1.85)$, II $8.83(2.19,0.82,1.89,2.35,1.58)$, III 8.57 (2.09, 0.77, $1.73,2.55,1.43)$, IV $11.22(2.65,0.82,2.55,3.62,1.58)$. Leg formula: 4123.

Carapace (Fig. 16) dark brown; median furrow black. Both eye rows (Fig. 17) procurved in dorsal view. Clypeus brown. Chelicerae brown, distally yellowish-brown. Endites yellowish. Labium triangular and brown, distally white. Sternum yellowishbrown and furnished with sparse black setae, lateral margin with small and pointed extensions fitting in coxal concavities of legs. Legs light yellowish-brown except femora that are light brown; metatarsi II-IV distally with ventral hair tufts. Opisthosoma (Fig. 16) oval, longer than wide, dorsally black, with dense hairs and three pairs of white 


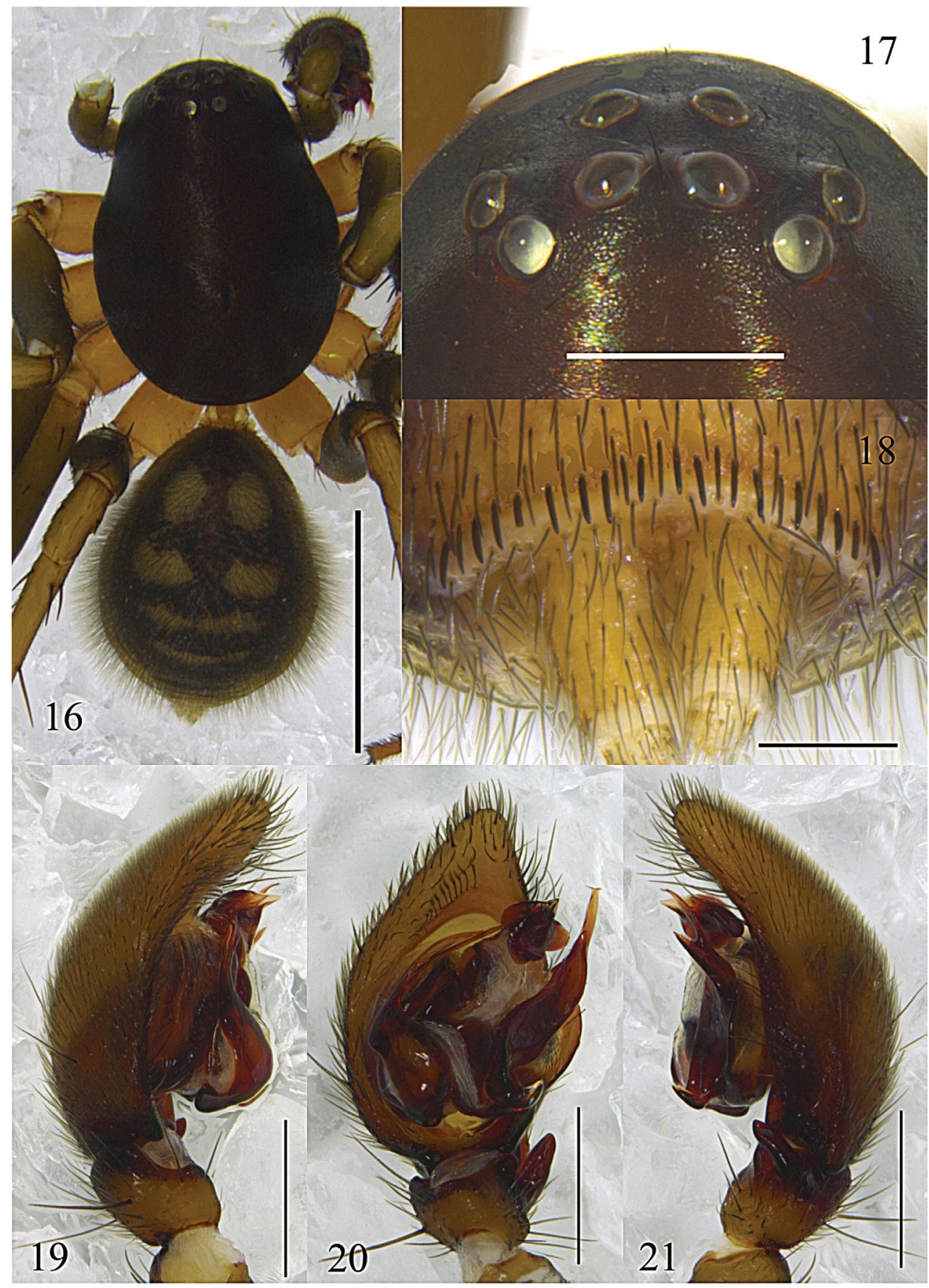

Figures 16-21. Mallinella pluma sp. n., 16 male habitus, dorsal view 17 ocular area, frontal view 18 male, posterior ventral spines, ventral view 19 left male palp, prolateral view $\mathbf{2 0}$ same, ventral view $2 \mathbf{I}$ same, retrolateral view. Scale bars: $2 \mathrm{~mm}$ (16); $0.2 \mathrm{~mm}(\mathbf{1 7}) ; 0.5 \mathrm{~mm}(\mathbf{1 8}-\mathbf{2 1})$. 


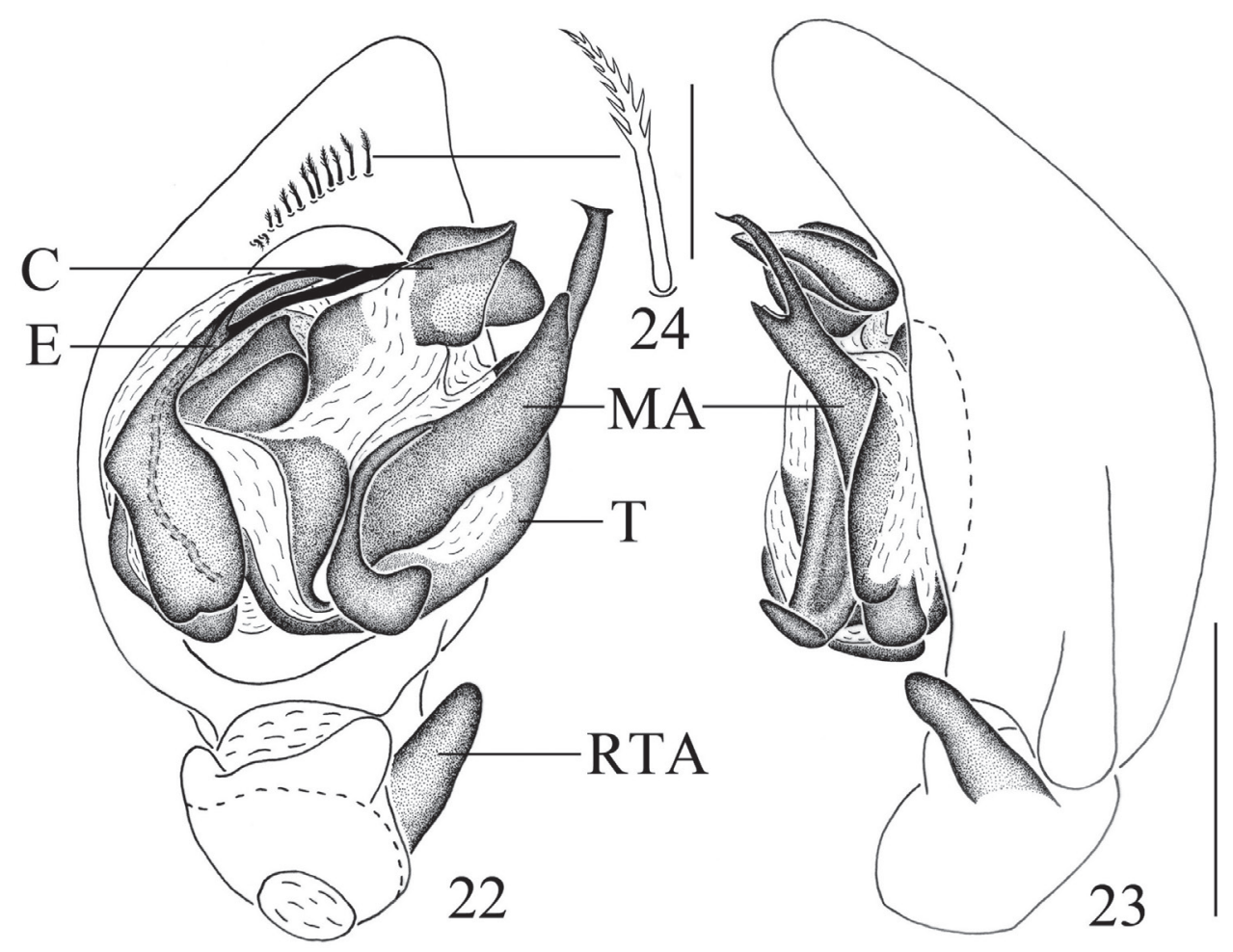

Figures 22-24. Mallinella pluma sp. n., 22 left male palp, ventral view 23 same, retrolateral view 24 a plumose hair (magnified). Scale bars: $0.5 \mathrm{~mm}$ (22-23); $0.05 \mathrm{~mm}$ (24).

lateral patches (the third slight connected), followed by one transverse white median patch; dorsal scutum indistinct; venter grey-black, covered with three irregular longitudinal white stripes; posterior ventral spines thin (Fig. 18), arranged in a single row. Spinnerets pale yellow.

Palpal organ (Figs 19-24). RTA digitiform, slightly wider at base, gradually tapering towards its blunt apex. Cymbium ventrally with a row of plumose hairs near its tip; cymbial fold broad, more than half as long as cymbium. Apex of median apophysis rostrated and pointing to the prolateral side; the median part of median apophysis with a small digitiform branch; baso-retrolateral fold narrow and long, spatulate, apex blunt. Conductor complex, with a triangular apophysis retrolaterally. Embolus bifurcated at the median part, lateral ramus shorter than mesal ramus, only lateral ramus with subterminal fold.

Female unknown.

Remarks. According to Dankittipakul et al. 2012, this species also should belong to sub-group 3 of the fronto species-group, but the plumose hairs on the cymbium ventrally makes that species unique; it is likely that $M$. pluma belongs to an unknown sub-group of the fronto species-group.

Distribution. China (Guangxi). 


\section{Acknowledgements}

We are grateful to Yingnan Wang for collecting the specimen from Guangxi. We are grateful to two anonymous referees for their valuable comments. This study was supported by the National Natural Science Foundation of China (No. 31071885, 30970325, 31093430), partly by the Education Office of Hebei Province, China (No. Z2009101) and the Natural Science Foundation of Baoding University (No. 2010Z01).

\section{References}

Dankittipakul P, Jocqué R, Singtripop T (2012) Systematics and biogeography of the spider genus Mallinella Strand, 1906, with descriptions of new species and new genera from Southeast Asia (Araneae, Zodariidae). Zootaxa 3369: 1-327.

Jocqué R (1991) A generic revision of the spider family Zodariidae (Araneae). Bulletin of the American Museum of Natural History 201: 1-160.

Platnick NI (2013) The world spider catalog, version 13.5. American Museum of Natural History, online at http://research.amnh.org/iz/spiders/catalog/INTRO1.html (accessed 3 January, 2013)

Song DX, Kim JP (1997) On seven new species of the family Zodariidae (Araneae) from China. Korean Arachnologica Arachnol 13(1): 7-17.

Song DX, Zhu MS, Chen J (1999) The Spiders of China. Hebei Science \& Technology Publishing House, Shijiazhuang, $640 \mathrm{pp}$.

Wang QB, Yin CM, Griswold C, Peng XJ (2009) Three new species of the genus Mallinela (Zodariidae: Araneae) from Yunnan Province, China. Zootaxa 2005: 47-56.

Wang QB, Yin CM, Peng XJ (2009) Description of Mallinella cymbiforma sp. nov. from Hunan Province, China (Zodariidae, Araneae). Acta Zootaxonomica Sinica 34: 14-17.

Wang XP, Ran JC, Chen HM (1999) A new species of Mallinella from China (Araneae, Zodariidae). Bulletin of the British Arachnological Society 11: 193-194.

Zhang BS, Zhu MS (2009) Three new species of the ant genus Mallinella Strand, 1906 (Araneae: Zodariidae) from China. Zootaxa 2212: 62-68.

Zhang F, Zhang BS, Jia XM (2012a) Two new species of the genus Mallinella Strand, 1906 (Araneae: Zodariidae) from China. Zootaxa 3241: 64-68.

Zhang F, Zhang BS, Jia XM (2012b) Two new species of the ant spider genus Asceua Thorell, 1887 (Araneae: Zodariidae) from China. Zootaxa 3307: 62-68. 